\title{
Das duktale Carcinoma in situ der Mamma
}

\author{
J. Pfisterer \\ Klinik für Gynäkologie und Geburtshilfe an der Christian-Albrechts-Universität Kiel
}

Das duktale Carcinoma in situ (DCIS) ist eine Läsion, deren Zellen morphologisch die typischen Veränderungen eines Karzinoms aufweisen, wobei diese jedoch nur innerhalb des duktalen Systems der Brustdrüse anzutreffen sind, eine Invasion ins umgebende Stromagewebe liegt noch nicht vor. Somit gilt es als noch nicht metastasierungsfähige Vorstufe invasiver Karzinome. Trotz dieser klaren Definition stellt das DCIS eine sehr heterogene Gruppe von Läsionen dar. Diese Heterogenität betrifft die Histopathologie, die Klinik und nicht zuletzt die Prognose.

Noch Anfang der 80er Jahre umfaßte das DCIS lediglich etwa $2 \%$ aller Brustkrebsfälle, heute liegt die Rate bei mehr als $12 \%$. Ursache hierfür ist der zunehmende Einsatz des Mammographie-Screenings mit dem Leitsymptom «Mikrokalk». Während der Anteil an DCIS an allen entdeckten Karzinomen bei etwa $3-5 \%$ liegt, macht er bei Screeningkollektiven inzwischen $20-30 \%$ aus. Aus historischen und teilweise unbehandelten Fällen weiß man, daß etwa $30 \%$ der Patientinnen mit einem DCIS ohne Therapie ein invasives Karzinom bekommen, das Zeitintervall liegt zwischen 3 und 10 Jahren (Mittel: 6,1 Jahre).

Erst durch die Mammographie ist es, überwiegend durch Nachweis von Mikroverkalkungen, möglich, ein DCIS bei einer asymptomatischen Patientin zu erkennen. Somit bedeutet die Mammographie Früherkennung mit der Chance auf Heilung im eigentlichen Sinn. Sonographie und MR-Mammographie spielen bei der Erkennung des DCIS nur eine untergeordnete Rolle.

Die Mastektomie stellt beim DCIS für 98\% aller Fälle eine kurative Behandlung mit definitiver Heilung dar (Tab. 1). Auch heute noch müssen sich alle anderen Therapieverfahren an diesem Standard messen [1]. Eine alleinige Exzision ist mit einer relativ hohen (bis $43 \%$ ), auch invasiven (bis 50\%) Rezidivrate verknüpft. Die Exzision mit anschließender Strahlentherapie senkt die Rezidivrate deutlich $(<20 \%)$, dennoch gibt es auch hier invasive Rezidive ( 50\%) [1]. Die mittlere Zeit bis zum Auftreten des intramammären Rezidivs liegt bei über 5 Jahren. Tritt dann ein nichtinvasives Rezidiv auf, das mit einer Mastektomie behandelt wird, sind alle Patientinnen nach 5 Jahren tumorfrei. Bei invasiven Rezidiven müssen wir von $10 \%$ Todesfällen nach 5 Jahren ausgehen. Bislang waren keine sicheren prädiktiven Faktoren für die Therapiewahl etabliert.
Die Vorhersage des Rezidivs war ebensowenig möglich wie die Art des Rezidivs (invasiv vs. nichtinvasiv). In den letzten Jahren wurde versucht, über neuere Klassifikationssysteme diese Problematik zu lösen. So haben verschiedene Arbeitsgruppen unterschiedliche Klassifikationssysteme entwickelt, wobei die von Silverstein und Mitarbeitern [2] publizierte Klassifizierung mit am bekanntesten ist: Hierbei werden drei Gruppen gebildet: Gruppe 1 besteht aus den «non high nuclear grade»-Fällen ohne Nekrose, Gruppe 2 aus den «non high nuclear grade»Fällen mit Nekrose und Gruppe 3 aus den «high nuclear grade»-Fällen. Hierauf baut der Van-Nuys-Prognostic-Index (VNPI) auf, wobei neben der pathologischen Klassifizierung, welche entsprechend der jeweiligen Gruppe mit 1 bis 3 Punkten bewertet wird, die Tumorgröße sowie der tumorfreie Resektionsrand Eingang finden. Es können prinzipiell Punktwerte von minimal 3 bis maximal 9 erreicht werden. Wiederum werden drei Klassen gebildet (VNPI 3 oder 4, VNPI 5-7, VNPI 8 oder 9), die sich - zumindest am retrospektiven Untersuchungsgut ausgewertet - prinzipiell voneinander unterscheiden: Die Niedrigrisikogruppe (VNPI 3 oder 4) weist nur etwa $2 \%$ Rezidive auf, die 8 Jahre rezidivfreie Überlebenszeit (RFS) liegt bei $97 \%$. In der mittleren Gruppe (VNPI 5-7) liegt die Rezidivrate bei $19 \%$, die 8 Jahre-RFS liegt bei $77 \%$, in der Hochrisikogruppe (VNPI 8 oder 9) beträgt die Rezidivrate $57 \%$, die 8-Jahre-RFS liegt bei nur 20\%. Darüber hinaus scheint es möglich, Patientinnen, die von einer Strahlentherapie profitieren, mit dem VNPI identifizieren zu können: In der Niedrigrisikogruppe beträgt die 8-Jahre-RFS mit Strahlentherapie $100 \%$, ohne Strahlentherapie $97 \%$, in der mittleren Gruppe liegen die Werte bei 88 versus 71\%, in der Hochrisikogruppe bei 35 versus $0 \%$. Bislang ist der VNPI jedoch nie prospektiv validiert worden.

Kürzlich konnte von Silverstein und Mitarbeitern [3] gezeigt werden, daß die Größe des tumorfreien Randsaumes bei der Exzision der entscheidende Parameter für das Risiko der Rezidiventstehung ist. Bei Patientinnen mit einem Randsaum von $>10 \mathrm{~mm}$ scheint unabhängig vom VNPI ein Verzicht auf eine Strahlentherapie möglich. Nur Patientinnen mit einem chirurgischen Randsaum von weniger als $1 \mathrm{~mm}$ sollen von einer Strahlentherapie profitieren (Tab. 2).

Auch in der Prävention einer nichtinvasiven Brustkrebserkrankung gibt es neue Erkenntnisse [4, 5]: So konnte gezeigt

\begin{tabular}{ll}
\hline KARGER & ( ) 1999 S. Karger GmbH, Freiburg \\
Fax +49761 4520714 & Accessible online at: \\
$\begin{array}{l}\text { E-mail kargergmbh@aol.com } \\
\text { www.karger.com }\end{array}$ & http://BioMedNet.com/karger \\
\end{tabular}

PD Dr. med. Jacobus Pfisterer

Klinik für Gynäkologie und Geburtshilfe an der Christian-Albrechts-Universität Michaelisstraße 16, D-24105 Kiel (Deutschland) Tel. +49 4315974089 , Fax -5974090 E-mail jpfisterer@email.uni-kiel.de 
Tab. 1. Rezidive nach DCIS-Metaanalyse 1999 [modifiziert nach 1]

\begin{tabular}{llcc}
\hline & $\mathrm{n}$ & Rezidive (\%) & $95 \%$ CI \\
\hline OP & 1148 & 22,5 & $16,9-28,2$ \\
OP + RT & 1452 & 8,9 & $6,8-11,0$ \\
Mastektomie & 1574 & 1,4 & $0,7-2,1$ \\
\hline
\end{tabular}

$\mathrm{CI}=$ Konfidenzintervall; $\mathrm{OP}=$ brusterhaltende Operation; $\mathrm{RT}=$ Radiotherapie

Tab. 2. DCIS - Rezidive [modifiziert nach 3]

\begin{tabular}{lllll}
\hline $\begin{array}{l}\text { Tumorfreier } \\
\text { Randsaum }\end{array}$ & $\mathrm{OP}^{1}$ & $\mathrm{OP}+\mathrm{RT}^{1}$ & $\mathrm{RR}$ & $\mathrm{p}$ \\
\hline$\geq 10 \mathrm{~mm}$ & 0,03 & 0,04 & 1,14 & 0,92 \\
$1-9 \mathrm{~mm}$ & 0,20 & 0,12 & 1,49 & 0,24 \\
$<1 \mathrm{~mm}$ & 0,58 & 0,30 & 2,54 & 0,01 \\
\hline
\end{tabular}

$\mathrm{RR}=$ relatives Risiko

${ }^{1}$ Rezidivwahrscheinlichkeit innerhalb 8 Jahren.

Tab. 3. Prävention-1 [modifiziert nach 4]

\begin{tabular}{llrll}
\hline \multirow{2}{*}{ Typ BRCA } & $\begin{array}{l}\text { Typ } \\
\text { Brusterkrankung } \\
\text { anamnestisch }\end{array}$ & \multicolumn{2}{c}{ Rate pro 1.000 Frauen } & \multirow{2}{*}{ RR } \\
\cline { 3 - 4 } & & Placebo & TAM & \\
\hline invasiv & LCIS nein & 6,45 & 3,30 & 0,51 \\
invasiv & LCIS ja & 12,99 & 5,69 & 0,44 \\
invasiv & ADH nein & 6,48 & 3,61 & 0,56 \\
invasiv & ADH ja & 10,11 & 1,43 & 0,14 \\
nichtinvasiv & LCIS nein & 2,09 & 1,02 & 0,49 \\
nichtinvasiv & LCIS ja & 12,72 & 7,26 & 0,57 \\
nichtinvasiv & ADH nein & 2,60 & 1,26 & 0,49 \\
nichtinvasiv & ADH ja & 3,09 & 2,39 & 0,78 \\
\hline
\end{tabular}

BRCA = Brustkrebserkrankung; $\mathrm{RR}=$ relatives Risiko; TAM = Tamoxifen; LCIS = lobuläres Carcinoma in situ; $\mathrm{ADH}=$ atypisch proliferierende duktale Hyperplasie.

werden, daß eine Tamoxifengabe nicht nur das Risiko für eine nichtinvasive Brustkrebserkrankung um etwa die Hälfte senken kann, sondern daß auch bei vorausgehendem lobulärem Carcinoma in situ und bei vorausgehender atypisch proliferierender duktaler Hyperplasie das Risiko einer nachfolgenden nichtinvasiven Brustkrebserkrankung deutlich reduziert wird (Tab. 3).
Sollten sich die neuesten Forschungsergebnisse auch in weiteren Untersuchungen bestätigen lassen, so stehen sowohl im operativen als auch im präventiven Bereich grundlegende Veränderungen an: Zum einen ist es dann erstmals möglich, das Risiko auch für eine nichtinvasive Brustkrebserkrankung durch eine medikamentöse Vorsorge deutlich senken zu können, zum anderen gewinnt die chirurgische Strategie für das weitere rezidivfreie Überleben der Patientin weiter an Bedeutung.

Daher müssen Pathologen und Kliniker umfangreiche Anforderungen erfüllen, damit die für eine Brusterhaltung geeigneten Fälle von den hierfür nicht geeigneten unterschieden werden können: So muß eine metrische Bestimmung der Läsion in drei Dimensionen auch bei makroskopischer Nicht-Abgrenzbarkeit gewährleistet sein, bei einer invasiven Komponente muß diese semiquantitativ von der nichtinvasiven ausgewertet werden. Von überragender Bedeutung ist die Metrik des minimal tumorfreien Abstandes zwischen Läsion und chirurgischem Resektionsrand in allen Ebenen. Vom Kliniker muß die vollständige Entfernung der Läsion, möglichst in einem operativen Schritt, gefordert werden. War das Leitsymptom Mikrokalk, ist neben einem Präparatradiogramm die Anfertigung einer postoperativen Mammographieaufnahme unerläßlich. Bei brusterhaltender Therapie ist ein intensives Follow-up der betroffenen Brust mit 1/2jährlichen Mammographiekontrollen für 4 Jahre und später jährlichen Kontrollen erforderlich.

Falls irgend möglich, sollte eine brusterhaltende Therapie angestrebt werden. Die Rezidivrate innerhalb von 10 Jahren liegt bei etwa $10-15 \%$. Ungefähr die Hälfte der Rezidive sind invasiv (5-7\%). Das Risiko «Tod durch Brustkrebs» liegt etwa bei $1 / 3$ der Erkrankten (2-3\%). Das Risiko «Tod durch DCIS/ invasives Karzinom nach Mastektomie wegen DCIS» liegt bei etwa $1-2 \%$. Insofern beträgt die Differenz in der Mortalitätsrate zwischen Brusterhaltung und Mastektomie beim DCIS 1-2\%. Möglicherweise kann diese Differenz durch neue präventive und/oder operative Strategien in Zukunft weiter verringert werden.

Zusammenfassend läßt sich festhalten, daß das DCIS eine Gruppe heterogener Läsionen mit unterschiedlich malignem Potential darstellt, daß eine Mastektomie nahezu 100\% Heilung bedeutet, daß man möglicherweise erstmals in der Lage ist, Fälle, die für eine alleinige brusterhaltende Operation in Frage kommen, von denen, die von einer zusätzlichen Strahlentherapie profitieren, unterscheiden zu können und daß man darüber hinaus auch die nicht für eine Brusterhaltung geeigneten Fälle identifizieren kann. Eventuell ist bei großzügigerer chirurgischer Resektion in fast allen Fällen der Verzicht auf eine Strahlentherapie möglich, darüber hinaus konnte die Effektivität einer medikamentösen Prävention erstmals nachgewiesen werden. Alle Verfahren setzen in jedem Fall neben der Aufklärung und Beratung der Patientin eine enge Zusammenarbeit zwischen Pathologen und Klinikern voraus. 


\section{Literatur}

1 Boyages J, Delaney G, Taylor R: Predictors of local recurrence after treatment of ductal carcinoma in situ. A meta-analysis. Cancer 1999;85:616-628.

2 Silverstein MJ, Lagios MD, Craig PH, Waisman JR, Lewinsky BS, Colburn WJ, Poller DN: A prognostic index for ductal carcinoma in situ of the breast. Cancer 1996;77:2267-2274.
3 Silverstein MJ, Lagios MD, Groshen S, Waisman JR, Lewinsky BS, Martino S, Gamagami P, Colburn WJ The influence of margin width on local control of ductal carcinoma in situ of the breast. N Engl J Med 1999;340:1455-1461.

4 Wickerham DL, Costantino J, Fisher B, Kavanah M, Wolmark N: Average annual rates of invasive and noninvasive breast cancer by history of LCIS and atypical hyperplasia for participants in the BCPT Proc ASCO 1999;327.
5 Fisher B, Dignam J, Wolmark N, Wickerham DL, Fisher ER, Mamounas E, Smith R, Begovic M, Dimitrov NV, Margolese RG, Kardinal CG, Kavanah MT, Fehrenbacher L, Oishi RH: Tamoxifen in treatment of intraductal breast cancer: National surgical adjuvant breast and bowel project B-24 randomised controlled trial. Lancet 1999;353:1993-2000. 